\title{
Synthetic Tooth Enamel: SEM Characterization of a Fluoride Hydroxyapatite Coating for Dentistry Applications
}

\author{
Marise Oliveira ${ }^{\mathrm{a} *}$, Herman Sander Mansur ${ }^{\mathrm{b} *}$ \\ Department of Metallurgical and Materials Engineering, Federal University of Minas Gerais, \\ Rua Espírito Santo, 35/206, 30160-030 Belo Horizonte - MG, Brazil
}

Received: August 16, 2006; Revised: April 12, 2007

\begin{abstract}
An alternative to etching enamel for retention of an adhesive is to grow crystals on the enamel surface. The potential advantages of crystal growth include easy procedure and less damage to the enamel. These crystals retain the adhesive or are the actual dental restoration. In this work, a paste of synthetic enamel was used to grow crystals of fluoride hydroxyapatite (F-HA) onto the human tooth surface. This technique can be used for several dentistry applications like enamel whitening, strengthening and restoration of early carie lesions. The low cost of reagents and simplicity of the technique along with the biocompatibility of the paste render possible the utilization on the market. The samples were prepared through the application of the paste by the incremental technique. The results obtained by scanning electron microscope (SEM)/EDX have indicated the deposition of a homogeneous layer of calcium phosphate that was grown onto the enamel substrate. The average thickness of the deposited film was in the range of 50-100 $\mu \mathrm{m}$ and with a similar density from the natural enamel observed by radiographic images.
\end{abstract}

Keywords: bioceramic, dentistry, biomaterial, hydroxyapatite, crystal growth, synthetic tooth

\section{Introduction}

The refinement of the technique of union of biomaterials to teeth has driven the development of several bonding agents to the enamel and currently it is the method of choice for the large majority of clinical dentists. This technique requires some form of mechanical anchoring between the enamel and the adhesive and therefore the dissolution of the external layer of the enamel by acid etching the tooth surface ${ }^{1}$. An alternative to the conventional acid etch of the dental enamel for the retention of an adhesive system is a revolutionary method of crystal growth on the surface of the enamel ${ }^{2}$. This technique is called "synthetic enamel" or crystals adhesion and, conventionally, it involves the application of a gel paste based on calcium/phosphate ions in acid medium onto the surface of the enamel, promoting the crystal growth directly from the tooth enamel inorganic structure ${ }^{3}$. As a result, the coating adheres firmly to the enamel for chemical retention and promotes a physical-chemical interconnection with the adhesive. A paste based on fluoride hydroxyapatite (F-HA) is prepared and applied onto the dental enamel and it promotes the formation and the growth of nanocrystals. In this procedure, the coating of the enamel by F-HA is a biomimetic process that involves the replication of the dental biological system. The F-HA coating presents the potential for the restoration of incipient carie lesions in dental enamel.

The introduction of the acid etching technique to the tooth enamel by Buonocore ${ }^{5}$ in 1955 gave a great improvement to the clinical procedures in Dentistry. This bonding technique needs some mechanical adhesion and consequently has disadvantages including enamel loss of the outer layer of the tooth ${ }^{4}$. Considerable research efforts have been made to develop bonding materials that help preserve enamel and it is clear that the most effective and reliable adhesives available at present are the composites. However, they are technique sensitive and the color stability of these materials are yet unsatisfactory ${ }^{4,5}$. An alternative to the use of composites is the biomimetic process. Biomimetism means that information or features of a biological system are implemented in an artificial one. It may be applied to numerous strategies in research and development of biomaterials. It means that one can optimize the mechanical properties of the material bulk by mimicking the natural structure of tissues ${ }^{6}$. The concept of biomimesis has long been used in chemistry in the context of compounds with enzyme-like catalytic action. Since the mid-1980 seconds it has been applied to materials, particularly with a view to producing ceramic and composite materials with improved toughness, analogous to shell, tooth, and bone. There was some sort of distress in the materials community with the idea of mimicking biological materials in the sense of producing an undistinguishable copy.

Hydroxyapatite (HA) is the main biomineral component of human hard tissues (tooth and bone) and its stoichiometry is represented by the formula $\mathrm{Ca}_{10}\left(\mathrm{PO}_{4}\right)_{6}(\mathrm{OH})_{2}{ }^{7-9}$. It is a biocompatible material when synthesized artificially as biomaterial and if fluoride ions substitute hydroxyl ions on the hexagonal unit cell, they give rise to fluorapatite$\mathrm{Ca}_{10}\left(\mathrm{PO}_{4}\right)_{6} \mathrm{~F}_{2}(\mathrm{FA})$. An apatitic mineral that incorporates less fluoride ions than the fluorapatite is considered to be a partial solid solution of fluoride into the HA structure (F-HA). Fluoride ions are incorporated into the apatite lattice through precipitation and growth reactions. This substitution brings about a reduction in the volume of the unit cell, so that the chemical stability of the apatite lattice is greatly enhanced by the electrostatic bond between fluoride and the adjacent ions ${ }^{10}$.

Associated with the biomimetic process, crystal growth can be used for dentistry applications on the enamel. Crystal growth conventionally involves the application of a sulphated acid solution to the enamel ${ }^{1,11-13}$, but at present a new acid solution and a F-HA paste is used. The result is a layer of F-HA crystals on the tooth enamel, suggesting a chemical interaction between the material and the tooth. This kind of bond is one of the main objectives of Dentistry and the material layer attained called synthetic enamel is similar in composition and density to the tooth enamel ${ }^{3}$. 
The aim of this study was to investigate the formation of the new synthetic bioceramic material and characterize some properties such as thickness and adhesion to the tooth enamel surface. In addition, the coating chemical composition, interface boundaries and morphologies were also analyzed.

\section{Experimental Procedure}

\subsection{Teeth sample preparation}

Six incisor teeth non carious, without any visual cracks or fractures extracted for orthodontic, periodontal and surgical reasons were used for synthetic enamel paste application. All the collected teeth were cleared of blood, epithelium and saliva by air-water spray and scrappers. After that, they were sterilized in auto-clave at $121^{\circ} \mathrm{C}$ for 15 minutes and were stored in distilled water at $4{ }^{\circ} \mathrm{C}$. All the teeth were treated by the same operator. This project was approved by Ethical Committee (UFVJM - Universidade Federal dos Vales do Jequitinhonha e Mucuri), under process number 020/05.

\subsection{F-HA crystal growth}

A new technique in which a white paste of modified hydroxyapatite which chemically resembles natural enamel is applied to the tooth surface. The paste is a mixture of hydrogen peroxide, phosphoric acid and F-HA powder. The hydroxyapatite (HA) was produced via the wet precipitation process as showed in Equation 1:

$$
\begin{aligned}
& 7 \mathrm{Ca}(\mathrm{OH})_{2 \text { (susp.) }}+3 \mathrm{Ca}\left(\mathrm{H}_{2} \mathrm{PO}_{4}\right)_{2} \cdot \mathrm{H}_{2} \mathrm{O}_{\text {(aq) }} \rightarrow \\
& \mathrm{Ca}_{10}\left(\mathrm{PO}_{4}\right)_{6}(\mathrm{OH})_{2} \downarrow+15 \mathrm{H}_{2} \mathrm{O}
\end{aligned}
$$

The $0.3 \mathrm{M} \mathrm{Ca}(\mathrm{OH})_{2}$ suspension and $0.12 \mathrm{M}$ calcium hydrogen phosphate hydrate $-\mathrm{Ca}\left(\mathrm{H}_{2} \mathrm{PO}_{4}\right)_{2} \cdot \mathrm{H}_{2} \mathrm{O}$ - solution were prepared at room temperature and vigorously stirred for 15 minutes. The $\mathrm{Ca}\left(\mathrm{H}_{2} \mathrm{PO}_{4}\right)_{2}$. $\mathrm{H}_{2} \mathrm{O}$ solution was added slowly to the $\mathrm{Ca}(\mathrm{OH})$, suspension and the mixture was stirred at room temperature for one hour. The resulting suspension was aged for 12 hours at room temperature and the supernatant was decanted ${ }^{9}$. The precipitate was subjected to vacuum filtering using filter paper $(9 \mu \mathrm{m})$ adapted in a Büchner funnel, washed 3 times with deionized water and filtered again. Then, the precipitate was dried at $110{ }^{\circ} \mathrm{C}$ for 24 hours. The amount of reagents was calculated to produce $10 \mathrm{~g}$ of HA. The paste was prepared by adding the HA powder to a $0.2 \mathrm{M} \mathrm{NaF}$ aqueous solution (Sol. B) under moderate stirring at $60{ }^{\circ} \mathrm{C}$ for 1 hour. The resulting gel-like paste of $\mathrm{F}^{-}$and HA was manually mixed with a solution of hydrogen peroxide at $35 \%$ (conc.) and phosphoric acid (conc. $>85 \%$ ) in 4:1 proportion respectively (Sol. A), forming a white viscous paste, named as F-HA paste (F-HA). The complete experimental procedure is illustrated on the flowchart showed in Figure 1. The paste application on the tooth enamel surface was preceded by covering the enamel with the solution A. In the sequence, the paste of F-HA was spread onto the enamel surface for 10 minutes as presented in Figure 2. A sequence of 8 applications was carried out on the same surface region in order to obtain a thick newly grown layer of F-HA. An aqueous fluoride solution $(0.48 \mathrm{M} \mathrm{NaF})$ was applied on the tooth surface at each application interval and at the end of the experiment.

\subsection{Characterization of the $F-H A$ growth layer and the powder of $F-H A$}

At first, the samples were submitted to the radiographic methods to verify the radiopacity in relation to the natural enamel in processed films, using conventional $\mathrm{x}$ ray equipment for daily clinical applications $(60-70 \mathrm{kV}, 5-10 \mathrm{~mA}, 100-500 \mathrm{~ms})$. The morphological characterization of sample surface by SEM (model JEOL JSM 6360 LV
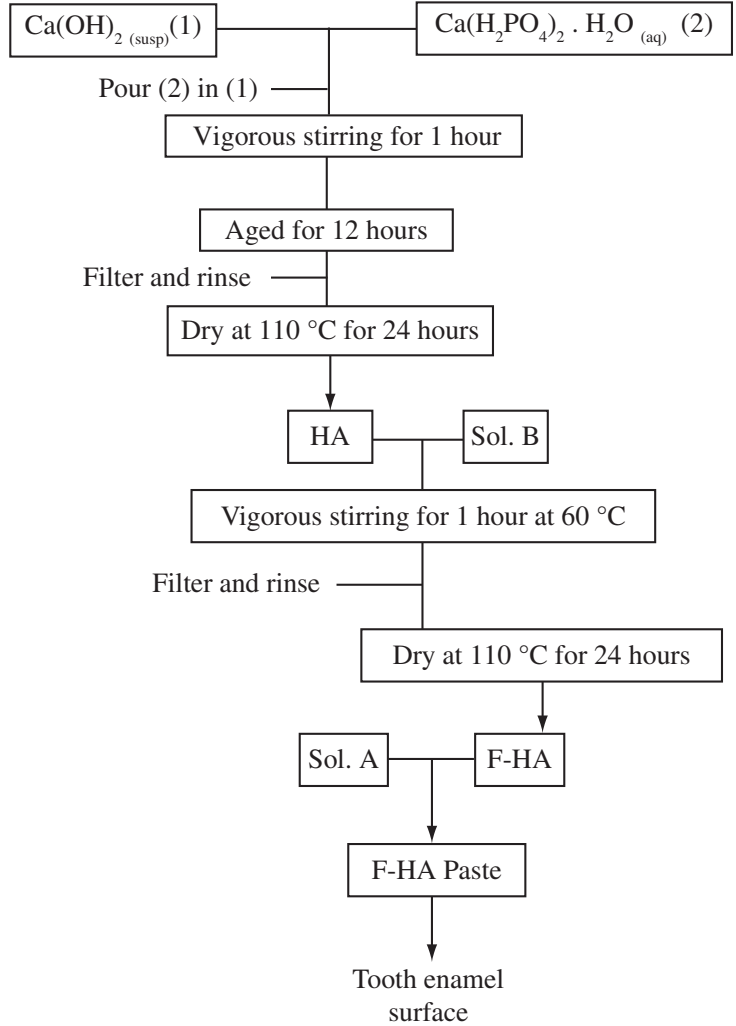

Figure 1. The flowchart of the procedure for producing F-HA paste.

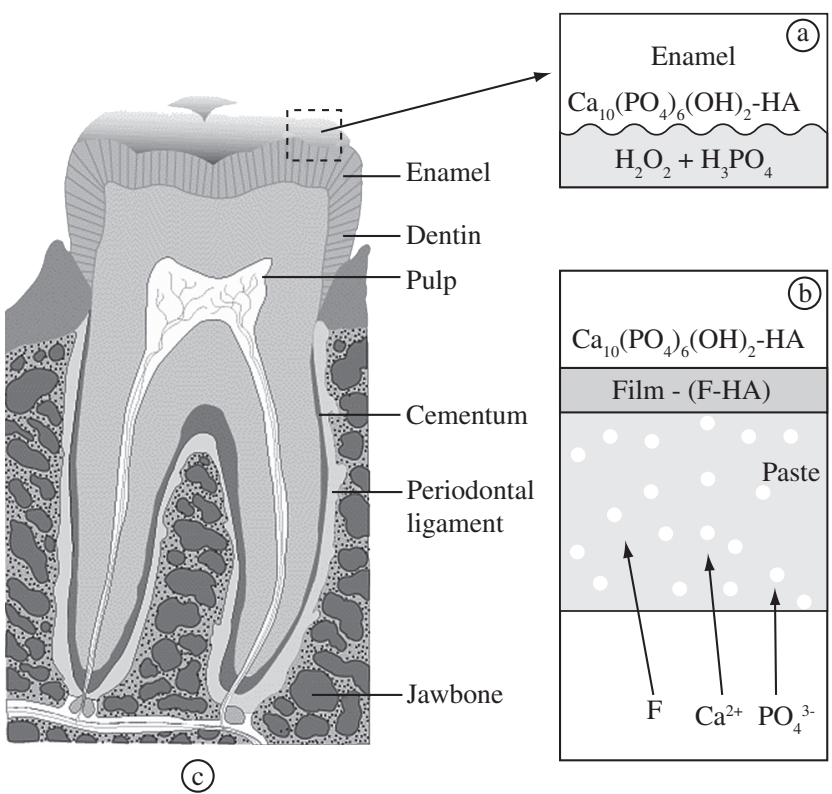

Figure 2. Schematic representation of the F-HA deposition onto tooth surface. a) The first step shows the dissolution of the enamel surface with solution-A; b) The layer film formation with F-HA paste; and c) Cartoon of Tooth tissue.

coupled with EDX microprobe) was carried out after performing the whole deposition procedure for F-HA growth onto the natural enamel. Before examination, samples were coated with a thin gold film by sputtering using low deposition rate, cooling of substrate and maximum distance between target and sample in order to avoid sample damage. Images of secondary electrons (SE) and backscat- 
tered electrons (BSE) were obtained using an accelerating voltage of $15 \mathrm{kV}$. The average thickness of the F-HA layer grown onto enamel was evaluated through measurements of samples cross-sections.

\section{Results and Discussion}

With this surface treatment technique, important synthesis parameters were modified to obtain a thicker calcium phosphate grown layer with simultaneous reduction on the deposition time.

The most probable reactions of the F-HA paste (Equation 2, Equation 3 and Equation 4) that are likely to occur are similar to those generally found on the conventional acid etch to the enamel as follows ${ }^{14}$ :

$$
\begin{aligned}
& \underbrace{\mathrm{Ca}_{10}\left(\mathrm{PO}_{4}\right)_{6}(\mathrm{OH})_{2(\mathrm{~s})}}_{\mathrm{HA}} \stackrel{2 \mathrm{H}^{+}}{\longrightarrow} 3 \mathrm{Ca}_{3}\left(\mathrm{PO}_{4}\right)_{2(\mathrm{~s})} \downarrow+ \\
& \mathrm{Ca}_{(\mathrm{aq})}^{2+}+\mathrm{H}_{2} \mathrm{O}+1 / 2 \mathrm{O}_{2} \uparrow \\
& \underbrace{\mathrm{Ca}_{3}\left(\mathrm{PO}_{4}\right)_{2(\mathrm{~s})}}_{\text {TCP }} \stackrel{2 \mathrm{H}^{+}}{\longrightarrow} 2 \mathrm{CaHPO}_{4} \downarrow+\mathrm{Ca}_{(\mathrm{Aq})}^{2+} \\
& 5 \mathrm{CaHPO}_{4}+\mathrm{H}_{2} \mathrm{O} \longrightarrow \mathrm{Ca}_{5}\left(\mathrm{PO}_{4}\right)_{3}(\mathrm{OH})_{(\mathrm{s})} \downarrow+ \\
& 2 \mathrm{H}_{3} \mathrm{PO}_{4}+\mathrm{H}_{2} \mathrm{O}
\end{aligned}
$$

Therefore, from Equation 2, the proposed reactions of fluorine with ename ${ }^{14}$ are presented in Equation 5:

$$
\begin{aligned}
& \mathrm{Ca}_{10}\left(\mathrm{PO}_{4}\right)_{6}(\mathrm{OH})_{2(\mathrm{~s})} \frac{\mathrm{HF}^{-} \mathrm{H}_{3} \mathrm{PO}_{4}}{\mathrm{H}_{2} \mathrm{PO}_{4^{-}}} \rightarrow \mathrm{Ca}_{10}\left(\mathrm{PO}_{4}\right)_{6} \mathrm{~F}_{2(\mathrm{~s})} \downarrow+ \\
& 2 \mathrm{OH}_{(\mathrm{Aq})}^{-} \text {(low } \mathrm{F} \text { concentration) }
\end{aligned}
$$


Figure 3. a) Dentistry radiographic image of the two incisor teeth shows that the layer formation onto the bucal surfaces of the teeth (arrows) using HA; Note: similar radiographic density of the natural enamel; and b) HA crystal structure adapted from? ${ }^{7}$.
The fluorine is the catalysts of the calcium phosphate formation, promoting the reactions of $\mathrm{Ca}^{2+}$ with $\mathrm{PO}_{4}{ }^{3-}$ even at reagent concentration up to 5 times lower ${ }^{14}$.

The results have indicated the deposition of a thicker layer on the oral surface of the tooth when compared to the others. Dentistry radiographic images revealed no significant difference on radiopacity between the new grown layer and tooth enamel suggesting a similar density as showed in Figure 3. The SEM photomicrograph from crosssection of the same sample (Figure 3 ) is presented in Figure 4.

A shiny surface (not showed) was observed after polishing, similar to the natural enamel. The F-HA deposited layer has adhered firmly to the tooth enamel surface, even when some mechanical stress for separation is applied or tooth fracture is performed in the samples, suggesting a strong chemical interaction at the interface. These results are in agreement with some previously similar reports in the literature $^{3}$, where synthetic apatite was used for rapid dental repair.

We have demonstrated that the investigated process can be useful by constructing "synthetic enamel" for dentistry applications. This synthetic F-HA paste can reconstruct enamel in a process that not only repairs early carie lesions but can also help to prevent their recurrence by strengthening the natural enamel with the fluorine presence ${ }^{3}$. Furthermore, other potential applications like whitening, where the paste promotes a whitening of the human tooth surface due to the use of hydrogen peroxide, orthodontic accessories adhesion by creating a layer that protect the enamel of HA dissolution and promoting a new surface of adhesion, and protection of the enamel at restoration cavity walls from recurrent of carie lesions and sealing of grooves, pits and fissures in deciduous and permanent teeth.

The HA synthesis via wet route was previously studied by our research group ${ }^{9}$ in order to produce and extensively characterize the several possibilities of obtaining calcium phosphates for biomedical application. In addition, some attention has been paid to the fact that it should be not only technically viable but also it has to be feasible to most common resources usually found in clinical and chemical 


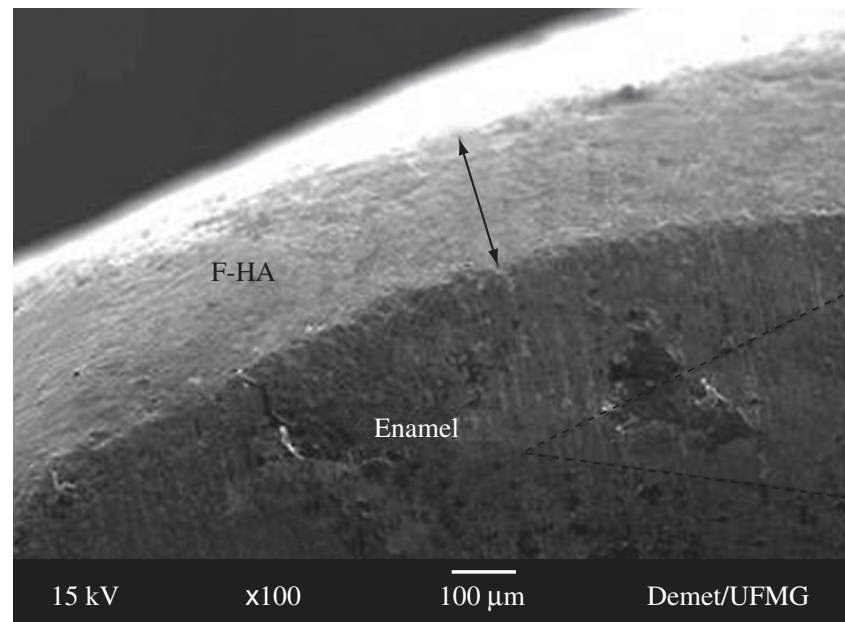

(a)

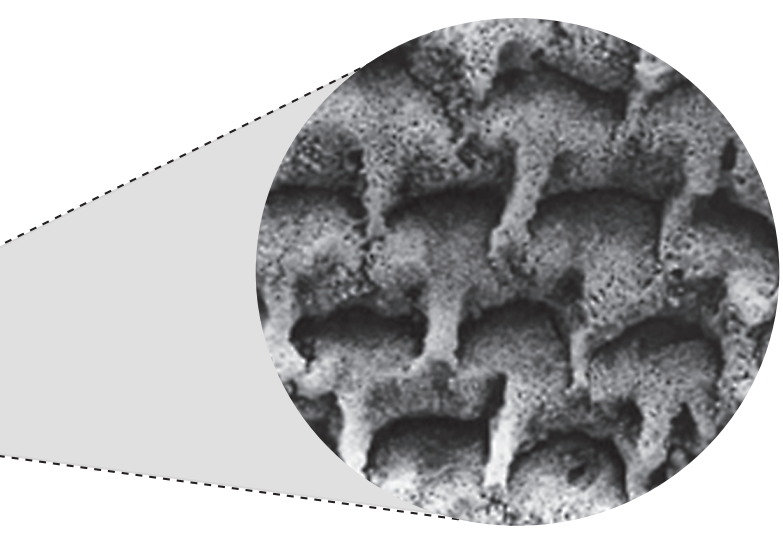

(b)

Figure 4. a) SEM image showing the thickness of the F-HA layer on the tooth bucal surface (arrow); and b) Insert detail: Adapted from?

laboratories ${ }^{9,15}$. Hence, by using the novel procedure developed in this study, where a synthetic enamel layer was grown onto tooth surface, a new window of opportunity is opened for daily clinical treatment of patients with carie lesions and also orthodontic needs.

\section{Conclusions}

A synthetic enamel layer was successfully obtained onto natural human tooth enamel tissue and extensively characterized by SEM/ EDX and radiopacity evaluation. The synthetic enamel was grown by using a paste produced by a combination of fluoride, HA, hydrogen peroxide in acid medium applied onto the natural enamel surface. Hence, the novel procedure developed in the present research has potential to be used in some important dentistry applications, for instance carie lesions treatment and orthodontics. We would like to emphasize that further investigation is under way for more in-depth characterization of the F-HA coating, regarding to its chemical composition, crystallinity and morphology at nano-scale level.

\section{Acknowledgments}

The authors would like to thank to Prof. Dagoberto Brandão Santos from Scanning Electron Microscopy Laboratory - School of Engineering/UFMG. Also, the authors acknowledge that the project was financially supported by CNPq/CAPES/FAPEMIG.

\section{References}

1. Maijer R, Smith DC. Crystal growth on the outer enamel surface. American Journal of Orthodontics. 1986; 89(3):183-193.

2. Powers JM, Messersmith ML. Enamel Etching and Bond Strength. In: Orthodontic Materials - Scientific and Clinical Aspects. Thieme Stuttgart (ed). New York, USA. 2001.

3. Yamagishi K, Onuma K, Suzuki T, Okada F, Tagami J, Otsuki M, Senawangse P. A synthetic enamel for rapid tooth repair. Nature. 2005; 433(7028):819-819.
4. Gorantla S, Valiathan A, Krishnan V K. An in vitro comparison of chitra polyurethane based radio-opaque light cured composite and a conventional composite as control. Trends of Biomaterials Artificial Organs. 2004; 18(1):46-51.

5. Buonocore MG. A simple method of increasing the adhesion of acrylic filling materials to enamel surfaces. J Dent Res. 1955; 34(6):849-853.

6. Blunk T, Gopferich A. Biomimetic polymers. Biomaterials. 2003; 24(24):4335-4335.

7. Jones FH. Teeth and bones: applications of surface science to dental materials and related biomaterials. Surface Science Reports. 2001; 42(3):75-205.

8. Legeros RZ. Properties of osteoconductive biomaterials: calcium phosphates. Clinical Orthopedics and Related Research. 2002; 1(395):81-98.

9. Santos MH, Oliveira M, Souza LPF, Mansur HS, Vasconcelos WL. Synthesis control and characterization of hydroxyapatite prepared by wet precipitation process. Materials Research. 2004; 7(4):625-630.

10. Aoba T, Shimazu Y, Taya Y, Soeno Y, Sato K, Miake Y. Fluoride and apatite formation in vivo and in vitro. Journal of Electron Microscopy. 2003; 52(6):615-625.

11. Artun J, Bergland S. Clinical trials with crystal growth conditioning as an alternative to acid-etch enamel pretreatment. American Journal of Orthodontics. 1984; 85(4):333-340.

12. Watts D C. Orthodontic Adhesive Resins and Composites: Principles of Adhesion. In: Orthodontic Materials - Scientific and Clinical Aspects. Thieme Stuttgart (ed). New York, USA. 2001.

13. Jones SP, Gledhill JR, Davies EH. The crystal growth technique - a laboratory evaluation of bond strengths. European Journal of Orthodontics. 1999; 21(1):89-93.

14. Tárzia O. Bioquímica Bucal. UFMG (ed), Belo Horizonte. 1991.

15. Oliveira, M. de. Synthesis and characterization based on Calcium Phosphate. Dissertation of Master in Metallurgical and Mining Engineering of UFMG, Belo Horizonte. 2004. 